\title{
PERCEPÇÃO DOS MORADORES DO BAIRRO LA ENCANTADA DE VILLA, LIMA, PERU SOBRE AS ÁREAS VERDES PÚBLICAS ${ }^{1}$
}

Jorge Mario Chávez Salas ${ }^{2}$, Alexandre França Tetto ${ }^{3}$, Carlos Augusto Reynel ${ }^{4}$, Nilton José Sousa ${ }^{5}$, Andressa Tres ${ }^{6}$

1 Parte dos estudos de doutorado do primeiro autor em Engenharia Florestal na Universidade Federal do Paraná, Curitiba - Brasil

2 Professor M.S. do Departamento Acadêmico de Manejo Florestal da Universidade

Nacional Agrária da Molina, Lima - Peru. E-mail: imchavez@lamolina.edu.pe

3 Professor Dr. do Departamento de Ciências Florestais, Universidade Federal do Paraná, Curitiba - Brasil.

4 Professor Ph.D. da Faculdade de Ciências Florestais, Universidade Nacional Agrária da Molina, Lima - Peru.

5 Professor Dr. do Departamento de Ciências Florestais, Universidade Federal do Paraná, Curitiba - Brasil.

6 Doutoranda em Engenharia Florestal. Universidade Federal do Paraná, Curitiba - Brasil.

Recebido em: 04/10/2019 - Aprovado em: 30/11/2019 - Publicado em: 15/12/2019

DOI: 10.18677/EnciBio_2019B44

\section{RESUMO}

Numerosos estudos apontam a importância das áreas verdes públicas nas cidades. Porém, seus habitantes estão conscientes do seu valor? O que pensam as pessoas que têm espaços verdes privados em suas casas? Eles valorizam os espaços públicos? Uma pesquisa social quantitativa foi realizada sobre a percepção da comunidade do bairro La Encantada de Villa, em Lima, sobre a importância das áreas verdes e espaços públicos. Para isto, 241 questionários foram aplicados aos moradores, para identificar a percepção dos mesmos sobre parques públicos; preferências sobre as instalações e serviços; espécies de plantas preferidas para parques e procedimentos que devem ser desenvolvidos, para que os moradores cuidem dos parques e apoiem a sua administração. Espécies de árvores, arbustos e cobertura do solo resistentes às condições ambientais foram incluídos na pesquisa. Entre os resultados mais relevantes, destaca-se que mais de $90 \%$ dos moradores consideram que as áreas verdes de uso público são muito importantes ou bastante importantes, sendo as principais razões a necessidade de espaços recreativos para crianças e questões estéticas. Os moradores gostariam que as áreas verdes fossem tranquilas, com um espaço para as crianças, para corridas e exercícios e com serviços básicos, como banheiros e refeitório. Entre as espécies de árvores preferidas nos parques estão: Acacia saligna, Ficus carica, Coccoloba uvifera e Prosopis pallida. Foi mencionado que a melhor maneira de cuidar das áreas verdes é mantendo a limpeza e contribuindo mensalmente com uma taxa para manutenção. Pretende-se que os resultados sejam utilizados no planejamento de áreas verdes em La Encantada.

PALAVRAS-CHAVE: áreas verdes públicas, efetividade, manejo 


\title{
PERCEPTION OF THE RESIDENTS OF THE NEIGHBORHOOD OF LA ENCANTADA DE VILLA, LIMA, PERU ON THE PUBLIC GREEN AREAS
}

\begin{abstract}
Numerous studies point out the importance of public green areas in cities. However, are its inhabitants aware of its value? What do those people with private green spaces in their homes think? Do they value the public spaces? A quantitative social research was carried out on the perception of La Encantada de Villa community on the importance of green areas and public spaces. For this, 241 semi-structured questionnaires were applied to identify the perception about public parks; preferences about the services; species of plants preferred and procedures to be developed so the residents can take care of their parks and support their administration. Species of trees, shrubs and soil cover with resistance to the environment conditions were included in the research. It is highlighted that more than $90 \%$ of the residents consider that green areas of public use are very important or quite important, the main reasons being the need for recreational spaces for children and for aesthetic reasons. The residents would like the green areas to be peaceful, with a space for children, for running and exercises and with basic services such as restrooms and cafeteria. Among the tree species most preferred in the parks are Acacia saligna, Ficus carica, Coccoloba uvifera and Prosopis pallida. It was mentioned that the best way to support the green areas was to maintain cleanliness and contribute monthly with an economic fee. It is intended that the results could be used for the planning of green areas at La Encantada.
\end{abstract}

KEYWORDS: public green areas, effectiveness, management

\section{INTRODUÇÃO}

A presença de áreas naturais em contextos urbanos é cada vez mais importante para a qualidade de vida nas cidades, sendo reconhecidos como elementos centrais na promoção da sustentabilidade ambiental e da qualidade de vida nas cidades, por suas diversas funções sanitárias, sociais, culturais, estéticas, funcionais, econômicas ou ecológicas (MADUREIRA et al., 2018). Segundo as Nações Unidas (1996), as áreas verdes e a vegetação reduzem a contaminação do ar e criam condições microclimáticas mais adequadas, melhorando a qualidade de vida da população. O mesmo autor aponta que para essa melhoria, deve-se "desenvolver e apoiar a aplicação de melhores métodos de classificação territorial que integrem globalmente as necessidades contraditórias de solo urbano para a moradia, a indústria, o comércio, a infraestrutura, o transporte, espaços verdes e zonas arborizadas, considerando a necessidade de espaços para atividades cotidianas, como parques infantis, jardins, campos de esportes, zonas de recreação e terrenos adequados para a jardinagem e a agricultura urbana".

É importante destacar não só os benefícios ambientais que as áreas verdes podem proporcionar à população, mas também sua relevância social. Estas apresentam impacto positivo na qualidade de vida e na saúde da população, representando uma característica complexa e necessária da paisagem urbana (SCHÄFFLER; SWILLING, 2013). Como observado por Wolch et al. (2014), o acesso das pessoas aos espaços verdes é cada vez mais considerado como uma questão de justiça ambiental. Em um estudo realizado na Austrália, foi observado que tanto a quantidade de espaço verde quanto a qualidade contam para o bemestar na infância e, à medida que as crianças crescem, a importância do bem-estar torna-se mais relevante (FENG; ASTELL-BURT, 2017). É importante notar que as 
áreas verdes se constituem de diversas formas, desde praças, jardins, parques urbanos entre outros tipos, não podendo ser confundidas com conceitos distintos de áreas de lazer, como é o caso de clubes (GOMES RUBIRA, 2016).

Em primeiro lugar, deve ser analisado o que são as percepções. Calixto e Herrera (2010) assinalam que "as percepções resultam da necessidade que se tem para desenvolver em um ambiente determinado e fazer frente às exigências ou problemáticas da vida. As percepções não se encontram isoladas, intervêm diversas características, com as quais o sujeito se encontra convivendo em seu cotidiano, e percebe através dos sentidos, o que outros não atingem a perceber, pelo que é comum ver ou escutar o que de forma emocional queremos ou para o que estamos preparados, dado que a percepção não pode desconectar-se da personalidade, assim a pessoa interpreta dependendo das circunstâncias que vive e experimenta".

É importante ressaltar, segundo Aguilar et al. (2017), que "o estudo das percepções não configura um campo teórico e metodológico unificado, mas conforma-se por um conjunto de interesses interdisciplinares entre os quais concorrem as ciências naturais, antropológicas, psicológicas, geográficas, entre outras. Cada uma dessas ciências fornece ferramentas de análise para decifrar os aspectos centrais do interesse da pesquisa no estudo das percepções, especialmente para entender como os grupos humanos dão sentido ao ambiente $e$ como nos relacionamos com ele".

O objetivo principal deste estudo foi analisar a percepção dos moradores do bairro La Encantada, em Lima, a respeito da importância das áreas verdes como espaços de uso público em contraposição ao fato da maioria destes possuírem amplos jardins privados. Queria-se saber se, em bairros de alto nível econômico (A e B), como o de La Encantada, os moradores consideram que os parques públicos apresentam alguma importância dentro de suas prioridades. A hipótese do trabalho é de que os moradores de classes elevadas, por terem jardins privados e acesso a outras instalações, como clubes de campo, não valorizam a existência de parques públicos no seu bairro.

\section{Caracterização da área de estudo}

\section{MATERIAIS E MÉTODOS}

A pesquisa se desenvolveu no bairro de "La Encantada de Villa" (La Encantada), no distrito de Chorrillos, localizada ao sul da cidade de Lima, Peru (Figura 1), com moradores de nível socioeconômico A-B, com rendimentos por pessoa adulta que superam os U\$ 4304 dólares (nível A) e U\$2211 dólares (nível B) (APEIM, 2017). A área urbanizada está dividida em lotes de 1.250 ou $2.500 \mathrm{~m}^{2}$, apresentando uma área de urbanização de aproximadamente $900.000 \mathrm{~m}^{2}$ (GOOGLE EARTH, 2016) constituindo a moradia de cerca de 640 famílias. A maioria destes lotes possuem amplos jardins privados. $O$ bairro tem várias particularidades: está localizado ao lado do Oceano Pacífico; está muito perto da única área natural protegida da cidade de Lima (Refúgio de Vida Silvestre Pântanos de Villa); e possui muitos jardins e árvores em suas ruas, predominantemente palmeiras. $\mathrm{O}$ acesso ao bairro é controlado com portaria de controle/vigilância. $\mathrm{O}$ bairro tem pelo menos 10 terrenos cujas dimensões variam de 2.000 a $10.000 \mathrm{~m}^{2}$ disponíveis para os parques, embora são pouco cuidados ou mesmo abandonados. Somente um terreno tem sido trabalhado com o plantio de árvores e grama. Praticamente não tem parques habilitados para o uso público. Esse fato tem sido um dos principais motivos para se fazer a pesquisa: por que, se existem recursos, não há mais parques habilitados para o prazer geral? 


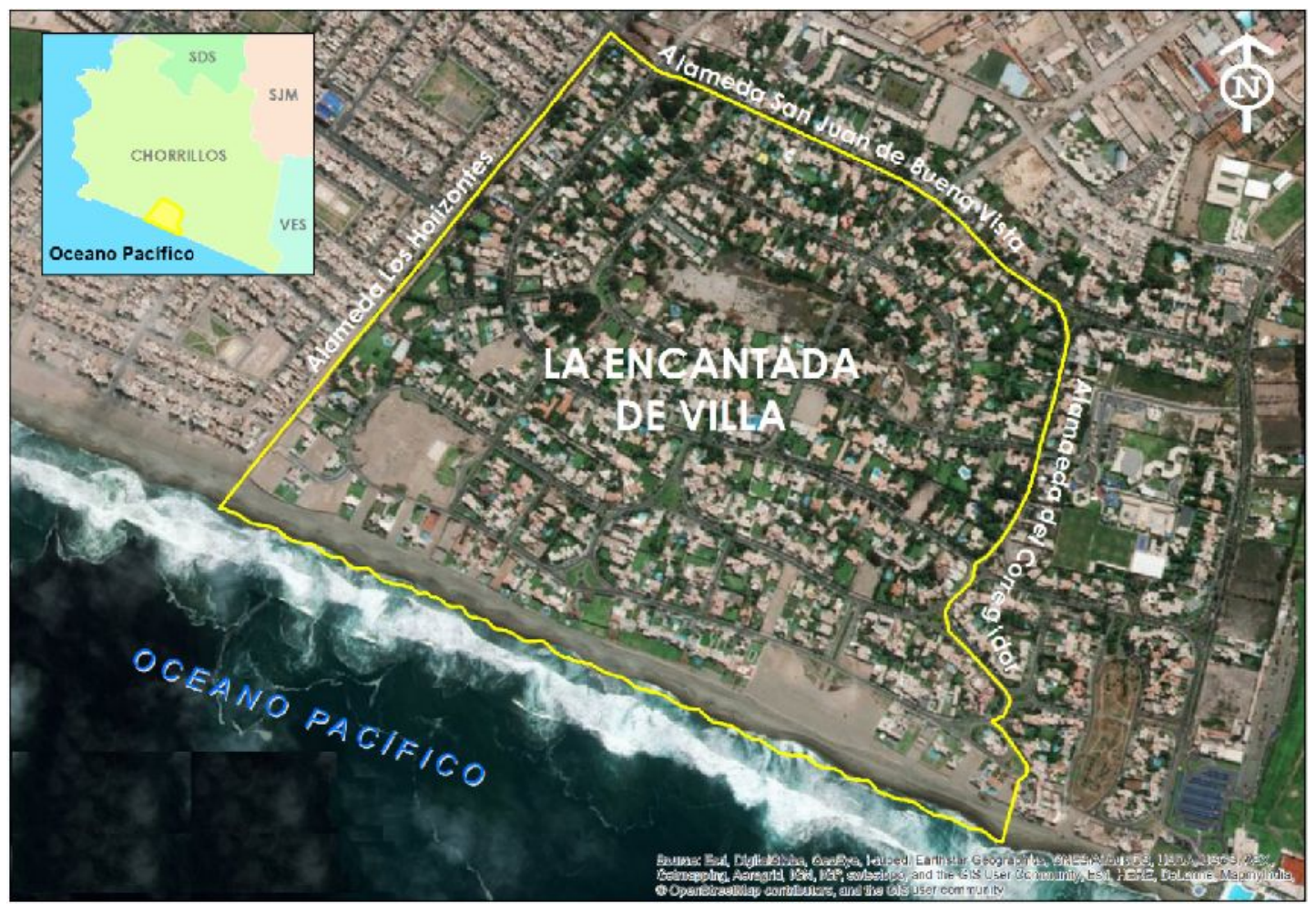

FIGURA 1 - Localização do Bairro La Encantada, Lima, Peru.

Fonte: Google Earth, 2016.

\section{Obtenção e análise de dados}

Foi realizada uma pesquisa social quali-quantitativa com 241 pessoas, por meio de aplicação de questionários diretos estruturados, onde as perguntas foram apresentadas da mesma forma e na mesma ordem para todos os entrevistados (INSTITUTO NACIONAL DE ESTADÍSTICA Y GEOGRAFÍA - MÉXICO, 2013). As questões foram agrupadas em tópicos relacionados às áreas verdes públicas. $O$ primeiro tópico tratou da percepção da importância das áreas verdes públicas para cada pessoa e para a comunidade, além da preferência por serviços que deveriam ser prestados nessas áreas, especialmente nos parques. O segundo tópico teve o objetivo de conhecer as opiniões sobre o tipo de vegetação (árvores, arbusto e cobertura do solo) preferida pelos moradores nas áreas verdes. O terceiro tópico refere-se à participação local para o cuidado das áreas, especificamente em relação a sugestões sobre as contribuições dos moradores para a manutenção das áreas verdes. Os três tópicos foram trabalhados de acordo com as faixas etárias e gênero dos entrevistados.

Para avaliar a percepção dos moradores do La Encantada sobre as áreas verdes públicas, foi utilizada a Análise de Conteúdo, que é um conjunto de técnicas usado para avaliar as percepções dos entrevistados, utilizando processos de categorização e tabulação de respostas a questões abertas (BARDIN, 1980). Para consultar as preferências dos moradores em relação aos três diferentes tipos de vegetação para os parques (árvores, arbustos e cobertura do solo), foram considerados os seguintes critérios: resistência à brisa do mar intensa e possíveis ventos fortes; capacidade de suportar intensa incidência solar em pelo menos quatro meses do ano, bem como temperaturas relativamente baixas (até 13 ${ }^{\circ} \mathrm{C}$ ) em três meses do ano; tolerância aos solos salinos e arenosos; e capacidade de suportar possíveis períodos de escassez de água doce para irrigação. 
As perguntas e respectivas alternativas de respostas foram:

1. De acordo com esta escala, qual o grau de importância em ter parques em sua comunidade? I - Importantíssimo; II - Bastante importante; III - Importante; IV - Pouco importante; V - Nada importante.

2. Por que é importante ou não ter parques em sua comunidade?

3. Assumindo uma área mínima de $10.000 \mathrm{~m}^{2}$, equivalente a um campo de futebol, que estrutura deveria incluir um novo parque em sua comunidade? Era possível indicar mais de uma alternativa: pista de corrida ou caminhada com distâncias marcadas, área de exercícios físicos (e. g. barras ou aparelho de abdominais), área para cães, área de jogos infantis, área de bancos sob a sombra de árvores, área de grama para exercícios físicos - espirituais (tipo yoga ou Tai Chi), área de elementos artísticos/arquitetônicos com plantas ornamentais, campo de futebol, pista de skate, lagoa com aves, pérgola ou zona para atuações ou exposições, quiosque ou lanchonete, banheiros, outro (mencione).

4. De acordo com esta escala, qual o grau de importância sobre ter diversos tipos de vegetação (árvores, arbustos, flores) nos parques de sua comunidade? I Importantíssimo; II - Bastante importante; III - Importante; IV - Pouco importante; V Nada importante.

5. Das árvores mostradas, que tipo de árvores preferiria que tenha o parque de sua comunidade? Mencione os de sua preferência (pode ser mais de um): algarrobo, Prosopis pallida H.et B. ex Willd; ficus/figueira, Ficus carica L.; huaranhuay, Tecoma sambucifolia H. et B.; meijo, Hibiscus tiliaceus L.; mimosa, Acacia saligna (Labill.) H. Wendel; mioporum, Myoporum laetum G. Forst; parkinsonia/pau-verde, Parkinsonia aculeata L.; tamarix, Tamarix aphylla (L.) Karst.; tara, Caesalpinia spinosa (Molina) Kuntze; e/ou uva-do-mar, Coccoloba uvifera L.

6. Das plantas ornamentais apresentadas, quais você prefere que existam no parque de sua comunidade? Mencione as de sua preferência (pode ser mais de um): abutilón, Abutilon sp.; buganville, Bougainvillea spectabilis Willd.; cactos-cereus, Cereus peruvianus (L.) Mill.; campanilla-azul, Convolvulus sabatius Viv.; heliotropo, Heliotropium arborescens L.; lantana-tricolor, Lantana camara L.; laurel-rosa, Nerium oleander L.; nandino, Nandina domestica Thumb.; retama, Spartium junceum L.; e/ou tecomaria, Tecoma capensis (Thumb.) Lindel.

7. Que tipo de cobertura apresentada prefere para a área do parque de sua comunidade? Mencione os de sua preferência (pode ser mais de um): grama artificial; grama natural, Stenotraphum secundatum (Walter) Kuntze; clavelillo, Dianthus deltoides L.; cóleus, Solenostemon sp.; lágrimas-da-virgem, Allium neapolitanum Cirillo; mariposita, Hypoestes phyllostachya Baker; portulaca, Portulaca grandiflora Hook; e/ou wedelia, Wedelia trilobata (L.) Hitchc.

8. De que forma os moradores podem colaborar com a manutenção e cuidado de um parque?

9. Tem algo a acrescentar ou sugerir em relação aos parques do La Encantada?

Os questionários foram aplicados pessoalmente às casas do bairro do dia 24 de setembro (sábado) ao dia 1 de outubro (sábado) do ano de 2016, das 10 às 19 horas. Posteriormente, os dados coletados pelos questionários foram organizados e tabulados por meio do programa Microsoft Office Excel® em planilhas eletrônicas, também sendo possível fazer a análise quantitativa e qualitativa das respostas dos entrevistados. Para as perguntas abertas, a análise e interpretação dos dados seguiu a metodologia de categorização temática proposta por Bardin (1980), onde as respostas contendo significados semelhantes dentro da frase, palavra isolada, ou 
palavras em conjuntos são agrupadas formando classes e, dessa forma, realiza-se a contagem de frequências de cada classe criada. Dada a variedade de respostas emitidas pelos entrevistados, foram anotadas as primeiras impressões dadas para serem posteriormente categorizadas e quantificadas.

Para a análise dos resultados, as respostas foram divididas por gênero e idade, tendo sido utilizadas as seguintes faixas etárias: de 15 a 19 anos, de 20 a 29 anos, de 30 a 39 anos, de 40 a 49 anos, de 50 a 59 anos e 60 anos ou mais. Os entrevistados foram classificados em faixas etárias, para descobrir as tendências das respostas das diferentes "gerações" sobre as áreas verdes. Por exemplo, se pessoas mais velhas apreciam mais espaços verdes públicos. Além disso, foi registrada a profissão do entrevistado.

Com base nas informações do número de famílias que vivem no La Encantada, o número médio de pessoas por família e as faixas etárias da área de estudo, foram definidas, para um nível de confiança de 95\% e erro amostral de 6,0\%, um total de 241 questionários de acordo com a amostragem para populações finitas.

\section{RESULTADOS E DISCUSSÃO}

Os resultados obtidos dos 241 questionários processados apresentam-se a seguir. A primeira questão a analisar é uma das mais importantes. Refere-se à opinião dos moradores sobre a importância das áreas verdes para o seu dia a dia. Esta é uma questão fechada onde só foi possível optar- por uma resposta. A Tabela 1 mostra as respostas dos entrevistados, agrupadas em idades e sexo.

TABELA 1 - Qual a importância de ter parques na comunidade? (\%)

\begin{tabular}{lrrrrrrrrr}
\hline \multicolumn{1}{c}{ Grau de } & \multicolumn{4}{c}{ Faixa etária (anos) } & \multicolumn{4}{c}{ Gênero } & Total \\
\cline { 2 - 9 } \multicolumn{1}{c}{ importância } & $15-19$ & $20-29$ & $30-39$ & $40-49$ & $50-59$ & $\geq 60$ & $q$ & $0^{\star}$ & \\
\hline Importantíssimo & 66,2 & 76,6 & 74,4 & 89,8 & 93,3 & 90,9 & 81,7 & 75,7 & 78,8 \\
Bastante importante & 15,4 & 19,1 & 10,3 & 6,1 & 6,7 & 0,0 & 11,9 & 11,3 & 11,6 \\
Importante & 18,5 & 4,3 & 15,4 & 2,0 & 0,0 & 9,1 & 6,3 & 12,2 & 9,1 \\
Pouco importante & 0,0 & 0,0 & 0,0 & 2,0 & 0,0 & 0,0 & 0,0 & 0,9 & 0,4 \\
Nada importante & 0,0 & 0,0 & 0,0 & 0,0 & 0,0 & 0,0 & 0,0 & 0,0 & 0,0 \\
\hline Entrevistados & 65 & 47 & 39 & 49 & 30 & 11 & 126 & 115 & 241 \\
\hline
\end{tabular}

* As diferenças entre idades foram significativas. As diferenças entre sexos não foram significativas.

Entre os entrevistados, 78,8\% atribuíram importância máxima (importantíssima), enquanto $11,6 \%$ atribuíram o segundo maior valor. Mais de $90 \%$ dos entrevistados afirmaram que os parques para a comunidade são importantíssimos ou bastante importantes. Por outro lado, cabe ressaltar que $0,4 \%$ dos entrevistados assinalou que os parques são pouco importantes, enquanto nenhum entrevistado os considerou nada importante. No trabalho de Menezes (2011), o resultado foi semelhante, visto que $100 \%$ dos entrevistados afirmaram ser extremamente necessário a presença de um parque próximo a suas casas, enquanto $6 \%$ mostraram pouco interesse na conservação de áreas verdes.

A mesma pergunta, processada segundo as idades, apresentou diferenças significativas entre as diferentes faixas de idades. Foi perceptível que os grupos de idades mais avançadas (40-49, 50-59 e 60 ou mais anos) atribuíram maior qualificação à importância dos parques para a comunidade, chegando a 89,8, 93,3 e $90,9 \%$ respectivamente. Os grupos de faixas etárias de 15 a 39 anos também atribuíram majoritariamente o valor máximo aos parques da comunidade, embora não em percentagens tão elevadas. A todos os que responderam que os parques 
eram "importantíssimos", foi realizada uma pergunta aberta a respeito da sua justificativa (Tabela 2).

TABELA 2 - Por que é "importantíssimo" ter parques na comunidade? (\%)

\begin{tabular}{|c|c|c|c|c|c|c|c|c|c|}
\hline \multirow[b]{2}{*}{ Respostas } & \multicolumn{6}{|c|}{ Faixa etária (anos) } & \multicolumn{2}{|c|}{ Género } & \multirow[b]{2}{*}{ Total } \\
\hline & $15-19$ & $20-29$ & $30-39$ & $\begin{array}{l}40- \\
49\end{array}$ & $\begin{array}{l}50- \\
59\end{array}$ & $\geq 60$ & 우 & $0^{x}$ & \\
\hline $\begin{array}{l}\text { Área de recreação para } \\
\text { crianças }\end{array}$ & 9,3 & 13,9 & 37,9 & 29,5 & 25,0 & 10,0 & 22,3 & 20,7 & 21,6 \\
\hline $\begin{array}{l}\text { Estética/beleza do lugar } \\
\text { Ambientes naturais }\end{array}$ & $\begin{array}{l}23,3 \\
23,3\end{array}$ & $\begin{array}{l}16,7 \\
22,2\end{array}$ & $\begin{array}{r}6,9 \\
17,2\end{array}$ & $\begin{array}{l}20,5 \\
11,4\end{array}$ & $\begin{array}{r}10,7 \\
3,6\end{array}$ & $\begin{array}{r}20,0 \\
0,0\end{array}$ & $\begin{array}{l}22,7 \\
13,6\end{array}$ & $\begin{array}{l}10,3 \\
17,2\end{array}$ & $\begin{array}{l}16,3 \\
15,3\end{array}$ \\
\hline $\begin{array}{l}\text { Lugar de encontro da } \\
\text { comunidade }\end{array}$ & 15,4 & 16,7 & 3,4 & 15,9 & 14,2 & 10,0 & 10,7 & 17,1 & 13,8 \\
\hline Entrevistados & 43 & 36 & 29 & 44 & 28 & 10 & 103 & 87 & 190 \\
\hline
\end{tabular}

Esta pergunta aberta permitiu aos entrevistados dar numerosas opiniões sobre a importância das áreas verdes públicas, mas decidiu-se mostrar as quatro respostas mais frequentes. Embora o bairro possua jardins privados abundantes e ruas com inúmeras árvores, principalmente palmeiras e jardins laterais, é o desejo dos moradores dispor de áreas onde possam aproveitar a natureza com segurança e integração com os vizinhos. Os maiores argumentos dos moradores para ter parques no bairro residem na necessidade de uma área de lazer para as crianças. Isso coincide com o apontado por Krellenberg et al. (2014). Estes autores observaram que os jovens usam os espaços verdes para fins de esporte ou para conhecer pessoas, enquanto os adultos e idosos preferem utilizá-los para passear, passear com crianças ou para contemplar a natureza.

Em segundo lugar ficou a resposta "estética/beleza do lugar", com maior percentagem de mulheres mencionando esta categoria, enquanto "áreas verdes" ocupou o terceiro posto, votado em segundo lugar pelos homens. A quarta e a quinta posições mencionadas pelos entrevistados de ambos os sexos foram "lugar de encontro para a comunidade" e "para entretenimento", ambos com 13,8 e 8,4\%. É importante observar que os entrevistados puderam fazer respostas múltiplas, em várias questões, e sugestões. Nos trabalhos de Menezes (2011) e Szeremeta e Zannin (2013) é possível verificar maior proporção de pessoas na busca por parques para as práticas de esportes e lazer principalmente. Algo que praticamente não foi mencionado pelos moradores, mas que Carrus et al. (2015) comentam em sua pesquisa, é que a presença de espaços verdes de alta qualidade dentro do contexto urbano promove a apreciação da natureza e promove atitudes mais positivas em relação à própria natureza, contribuindo para o objetivo global de tornar os cidadãos mais ambientalmente conscientes.

As faixas etárias 30-39, 40-49 e 50-59 anos indicaram como maior motivo porque são "áreas de recreação para as crianças", o grupo de idade de 15 a 19 anos como maior motivo a "estética do bairro". Finalmente, a faixa etária de 20 a 29 anos indicou como maior resposta o "ter áreas verdes na vizinhança". Foi surpreendente que praticamente não houve respostas sobre as áreas verdes reduzirem o estresse por meio de seu potencial restaurador que, como assinalam Martínez-Soto et al. (2016), representa um recurso importante para os habitantes que vivenciam continuamente cenários urbanos caóticos, como se manifesta na cidade de Lima. Os resultados deste artigo não corroboram com o trabalho apresentado por Madureira et al. (2015), realizado na França e Portugal, onde os entrevistados responderam em sua maioria sobre as vantagens das áreas verdes urbanas para "melhorar a saúde e o bem-estar" e "contato com a natureza". 
Das 14 opções de respostas sobre o que esperavam de parques públicos notou-se que os moradores do La Encantada preferem quatro elementos fundamentais que podem ser agrupados da seguinte maneira: ter uma área tranquila para aproveitar o ambiente, dispor de área de recreação para crianças, ter espaços para exercícios individuais e contar com serviços básicos como banheiros e lanchonetes. A Tabela 3 mostra as seis principais respostas feitas pelos moradores de La Encantada, separados por idade e sexo.

TABELA 3 - Elementos que deve conter um novo parque

\begin{tabular}{lrrrrrrrrr}
\hline \multicolumn{1}{c}{ Elementos } & \multicolumn{4}{c}{ Faixa etária (anos) } & \multicolumn{3}{c}{ Gênero } & \multirow{2}{*}{ Total } \\
\cline { 2 - 9 } & $15-19$ & $20-29$ & $30-39$ & $40-49$ & $50-59$ & $\geq 60$ & q & $0^{x}$ & \\
\hline Área de bancos sob a & 80,0 & 70,2 & 74,4 & 73,5 & 76,7 & 81,8 & 79,4 & 71,3 & 75,5 \\
sombra de árvores & 61,5 & 70,2 & 89,7 & 87,8 & 70,0 & 81,8 & 74,6 & 75,7 & 75,1 \\
Área de jogos infantis & 56,9 & 70,2 & 79,5 & 85,7 & 80,0 & 63,6 & 77,0 & 67,0 & 72,2 \\
Caminho de corrida ou & 56,9 & 68,1 & 76,9 & 69,4 & 60,0 & 63,6 & 65,9 & 65,2 & 65,6 \\
caminhada & 73,8 & 68,1 & 69,2 & 51,0 & 56,7 & 72,7 & 60,3 & 70,4 & 65,1 \\
Banheiros & 70,8 & 63,8 & 56,4 & 53,1 & 50,0 & 36,4 & 62,7 & 55,7 & 59,3 \\
Área de exercícios físicos & 65 & 47 & 39 & 49 & 30 & 11 & 126 & 115 & 241 \\
Quiosque ou refeitório & & & & & & & &
\end{tabular}

Pode-se observar que tanto os homens (segunda maior porcentagem) quanto as mulheres (maior porcentagem) optaram principalmente por "uma área de bancos sob a sombra das árvores". A partir disso se constatam dois fatos: a preferência dos usuários por árvores com objetivo de sombreamento, além de bancos para poder aproveitar a tranquilidade do parque.

Uma segunda resposta frequente foi o de ter área para crianças. Isto também pode ser relacionado ao conceito de utilizar o parque como espaço de reunião social, sendo as áreas para crianças uma das principais opções para a realização. Indiretamente, pode-se deduzir que os moradores preferem ter áreas verdes públicas onde os adultos e seus filhos possam se locomover amplamente e sem riscos, como por exemplo, quando há grama e não com plantas ornamentais que limitam os deslocamentos e jogos.

O caminho para corridas com distâncias marcadas foi selecionado em segundo lugar pelas mulheres entrevistadas, enquanto os homens colocaram em quarto lugar. Isto pode ser vinculado com a área de exercícios físicos, selecionados em sexto lugar pelas mulheres e em terceiro lugar pelos homens.

Os três grupos de idades intermediárias (20-29, 30-39 e 40-49 anos), que por dedução são os que devem ter crianças de pouca idade, privilegiaram a área de jogos infantis. Por outro lado, é grande o interesse dos grupos de 20 a 59 anos em uma área de corrida com distâncias marcadas, que diminui significativamente no grupo de 15 a 19 anos e de 60 anos ou mais. Infraestruturas como banheiros e lanchonete são bem recebidos pelos grupos de idades até 50 anos, não sendo considerados prioritários para os maiores de 50 anos. O fato dos moradores apresentarem preferências maiores para a sombra das árvores, em relação às demais instalações, coincide com os resultados obtidos por Madureira et al. (2018) que apontam que as instalações, como jogos infantis, quadras de esporte, cafés ou restaurantes são moderadamente apreciados.

A Tabela 4 apresenta as respostas relacionadas à importância de diversos tipos de vegetação nos parques. As duas respostas de maior relevância ("importantíssima" e "bastante importante") somaram $84,6 \%$, e se somado os que 0 consideram "importante", chega-se a $97 \%$ do total. É um bom indicativo do desejo 
dos moradores do La Encantada de possuir áreas verdes em seus espaços públicos. A diversidade de vegetação, isto é, árvores, arbustos e cobertura, é bem valorizada pela vizinhança, ao invés de possuir um só elemento, como a grama, por exemplo. Isso coincide com o que afirmam De La Barrera et al. (2016b), que estimam que quanto maior é a renda familiar no bairro, mais rica a estrutura da diversidade (por exemplo, árvores, arbustos e ervas) nos espaços verdes. Este é um tema que ainda precisa ser estudado mais profundamente.

TABELA 4 - Qual a importância de ter diversos tipos de vegetação nos parques da comunidade? (\%)

\begin{tabular}{lrrrrrrrrr}
\hline \multirow{2}{*}{ Grau de importância } & \multicolumn{4}{c}{ Faixa etária (anos) } & \multicolumn{3}{c}{ Gênero } & \multirow{2}{*}{ Total } \\
\cline { 2 - 9 } & $15-19$ & $20-29$ & $30-39$ & $40-49$ & $50-59$ & $\geq 60$ & \multicolumn{1}{c}{$\sigma^{\text {* }}$} & \\
\hline Importantíssimo & 56,9 & 51,1 & 64,1 & 81,6 & 73,3 & 81,8 & 67,5 & 62,6 & 65,1 \\
Bastante importante & 16,9 & 29,8 & 25,6 & 12,2 & 16,7 & 9,1 & 19,8 & 19,1 & 19,5 \\
Importante & 23,1 & 10,6 & 10,3 & 6,1 & 6,7 & 9,1 & 11,1 & 13,9 & 12,4 \\
Pouco importante & 3,1 & 8,5 & 0,0 & 0,0 & 3,3 & 0,0 & 1,6 & 4,3 & 2,9 \\
Nada importante & 0 & 0 & 0 & 0 & 0 & 0 & 0 & 0 & 0 \\
\hline Entrevistados & 65 & 47 & 39 & 49 & 30 & 11 & 126 & 115 & 241 \\
\hline
\end{tabular}

${ }^{*}$ As diferenças entre idades foram significativas. As diferenças entre sexos não foram significativas.

Embora as faixas etárias coincidam em dar a maior pontuação à afirmação que a existência de vários tipos de vegetação é importantíssima, o contraste de percentagens entre esses difere. Enquanto esta percentagem é de $81,6 \%$ e $81,8 \%$ nos grupos de 40-49 anos e de 60 anos ou mais, nas faixas etárias de 20 a 29 e de 15 a 19 anos é de 51,1 e 56,9\%, respectivamente. É válido notar que nenhum dos entrevistados respondeu que a existência de vários tipos de vegetação não é importante. Em relação à escolha de 10 espécies arbóreas mostradas aos entrevistados, quatro árvores se destacaram (Tabela 5).

TABELA 5 - Árvores de preferência para os parques da comunidade (\%)

\begin{tabular}{|c|c|c|c|c|c|c|c|c|c|}
\hline \multirow{2}{*}{ Espécies arbóreas } & \multicolumn{6}{|c|}{ Faixa etária (anos) } & \multicolumn{2}{|c|}{ Gênero } & \multirow{2}{*}{ Total } \\
\hline & $15-19$ & $20-29$ & $30-39$ & $40-49$ & $50-59$ & $\geq 60$ & + & $0^{x}$ & \\
\hline Mimosa & 44,6 & 51,1 & 41,0 & 59,2 & 50,0 & 27,3 & 50,0 & 46,1 & 48,1 \\
\hline Ficus & 38,5 & 46,8 & 43,6 & 51,0 & 56,7 & 36,4 & 42,1 & 49,6 & 45,6 \\
\hline Uva-do-mar & 53,8 & 38,3 & 43,6 & 44,9 & 40,0 & 36,4 & 44,4 & 45,2 & 44,8 \\
\hline Algarrobo & 27,7 & 57,4 & 43,6 & 44,9 & 43,3 & 45,5 & 40,5 & 44,3 & 42,3 \\
\hline Huaranhuay & 26,2 & 42,6 & 41,0 & 34,7 & 26,7 & 27,3 & 32,5 & 34,8 & 33,6 \\
\hline Tara & 27,7 & 46,8 & 23,1 & 34,7 & 13,3 & 36,4 & 34,1 & 27,0 & 30,7 \\
\hline Parkinsonia & 26,2 & 25,5 & 38,5 & 26,5 & 23,3 & 18,2 & 31,0 & 23,5 & 27,4 \\
\hline Meijo & 20,0 & 29,8 & 30,8 & 30,6 & 13,3 & 27,3 & 27,8 & 22,6 & 25,3 \\
\hline Mioporum & 16,9 & 23,4 & 25,6 & 22,4 & 23,3 & 9,1 & 20,6 & 21,7 & 21,2 \\
\hline Tamarix & 20,0 & 31,9 & 20,5 & 12,2 & 16,7 & 9,1 & 16,7 & 23,5 & 19,9 \\
\hline Eucalipto & 3,1 & 2,1 & 0,0 & 6,1 & 3,3 & 9,1 & 2,4 & 4,3 & 3,3 \\
\hline Sauc & 0,0 & 0,0 & 0,0 & 4,1 & 3,3 & 18,2 & 2,4 & 1,7 & 2,1 \\
\hline Molle-costeño & 0,0 & 0,0 & 2,6 & 6,1 & 3,3 & 0,0 & 4,0 & 0,0 & 2,1 \\
\hline Palmeiras & 0,0 & 6,4 & 0,0 & 2,0 & 3,3 & 0,0 & 0,8 & 3,5 & 2,1 \\
\hline Outros & 6,1 & 2,1 & 2,6 & 8,2 & 10,0 & 54,5 & 6,3 & 9,6 & 7,9 \\
\hline Entrevistados & 65 & 47 & 39 & 49 & 30 & 11 & 126 & 115 & 241 \\
\hline
\end{tabular}

A mimosa (Acacia saligna) foi a espécie que obteve maior preferência (primeira no caso de mulheres e segunda no caso dos homens), obtendo $48,1 \%$ das respostas. Neste caso, os entrevistados podiam indicar sua preferência por uma ou mais árvores. O ficus (Ficus carica) foi apresentado em segundo lugar no caso das mulheres e em primeiro lugar no caso dos homens, obtendo um percentual de $45,6 \%$. Visto que o questionário permitiu aos entrevistados propor outras espécies, 
algumas foram mencionadas, mas com percentagens que não superaram os 3,3\%: eucaliptos, sauces, molles e palmeiras (estas últimas exóticas, mas muito abundantes nas ruas da urbanização). É importante salientar a menção feita aos eucaliptos e sauces pelos entrevistados com mais de 60 anos, que não estavam incluídos nas imagens. Após a observação das figuras de 10 arbustos mostradas aos entrevistados, foi destacado como favorito, tanto por homens como mulheres, o bouganville, com $61,8 \%$ do total (Tabela 6 ).

TABELA 6 - Tipo de plantas e arbustos com flores de preferência para os parques de sua comunidade (\%)

\begin{tabular}{lrrrrrrrrr}
\hline \multirow{2}{*}{ Espécies arbustivas } & \multicolumn{4}{c}{ Faixa etária (anos) } & \multicolumn{2}{c}{ Gênero } & \multirow{2}{*}{ Total } \\
\cline { 2 - 9 } & $15-19$ & $20-29$ & $30-39$ & $40-49$ & $50-59$ & $\geq 60$ & $q$ & $0^{\star}$ & \\
\hline Bouganville & 56,9 & 74,5 & 56,4 & 65,3 & 56,7 & 54,5 & 62,7 & 60,9 & 61,8 \\
Heliotropo & 53,8 & 68,1 & 64,1 & 46,9 & 50,0 & 36,4 & 55,6 & 55,7 & 55,6 \\
Campanilla-azul & 55,4 & 63,8 & 56,4 & 49,0 & 36,7 & 18,2 & 53,2 & 50,4 & 51,9 \\
Retama & 40,0 & 44,7 & 56,4 & 55,1 & 56,7 & 63,6 & 50,0 & 49,6 & 49,8 \\
Laurel-rosa & 47,7 & 53,2 & 46,2 & 42,9 & 46,7 & 18,2 & 51,6 & 40,0 & 46,1 \\
Lantana-tricolor & 24,6 & 38,3 & 35,9 & 26,5 & 33,3 & 36,4 & 35,7 & 26,1 & 31,1 \\
Tecomaria & 26,2 & 31,9 & 25,6 & 32,7 & 30,0 & 9,1 & 25,4 & 31,3 & 28,2 \\
Abutilon & 26,2 & 31,9 & 28,2 & 16,3 & 16,7 & 18,2 & 26,2 & 21,7 & 24,1 \\
Nandina & 16,9 & 27,7 & 17,9 & 12,2 & 13,3 & 0,0 & 20,6 & 13,0 & 17,0 \\
Cactos-cereus & 13,8 & 19,1 & 17,9 & 22,4 & 10,0 & 0,0 & 13,5 & 19,1 & 16,2 \\
\hline Entrevistados & 65 & 47 & 39 & 49 & 30 & 11 & 126 & 115 & 241 \\
\hline
\end{tabular}

Em segundo lugar, em ambos os gêneros, foi mencionado o heliotropo (55,6\%), seguido pela campanilla-azul, a retama e o laurel-rosa com o 51,9, 49,8 e $46,1 \%$, respectivamente. As outras espécies propostas tiveram respostas de $31,1 \%$ a 16,2\%. Deduz-se que o favoritismo pelo bouganville se deve a seu caráter de planta trepadeira, o que favorece muito a sombra em lugares com bancos, além de possuir flores muito populares em toda a cidade de Lima.

A análise de resultados das preferências dos moradores em relação às plantas e arbustos com flores para seus parques, de acordo com as faixas etárias, também mostrou um destacado favoritismo para o boungaville. Quatro das seis faixas etárias, com exceção dos grupos de 30 a 39 anos e de 60 anos ou mais, deram-lhe a máxima percentagem.

Quanto à cobertura do solo dos parques, dentre as oito alternativas propostas, destacou-se a preferência, tanto de homens como de mulheres, para a grama, ocupando o primeiro lugar, com $88 \%$ das respostas (Tabela 7 ). Isso reforça o que é obtido na Tabela 3 , onde a maioria das pessoas procuram um local público para se encontrar com os vizinhos e as crianças brincarem, sendo a grama a vegetação que permite maior mobilidade para pessoas de todas as idades.

TABELA 7 - Tipos de coberturas de preferência para os parques de sua comunidade (\%)

\begin{tabular}{lrrrrrrrrr}
\hline \multicolumn{1}{c}{ Tipos de cobertura } & \multicolumn{4}{c}{ Faixa etária (anos) } & \multicolumn{3}{c}{ Gênero } & \multirow{2}{*}{ Total } \\
& $15-19$ & $20-29$ & $30-39$ & $40-49$ & $50-59$ & $\geq 60$ & + & $o^{*}$ & \\
\hline Grama & 86,2 & 93,6 & 87,2 & 85,7 & 93,3 & 72,7 & 86,5 & 89,6 & 88,0 \\
Coleus & 15,4 & 25,5 & 23,1 & 28,6 & 40,0 & 18,2 & 23,8 & 25,2 & 24,5 \\
Wedelia & 24,6 & 29,8 & 30,8 & 20,4 & 6,7 & 27,3 & 20,6 & 27,0 & 23,7 \\
Lágrimas da virgem & 20,0 & 25,5 & 25,6 & 24,5 & 23,3 & 27,3 & 26,2 & 20,9 & 23,7 \\
Clavelillo & 18,5 & 17,0 & 15,4 & 20,4 & 23,3 & 9,1 & 18,3 & 18,3 & 18,3 \\
Portulaca & 13,8 & 21,3 & 7,7 & 18,4 & 16,7 & 27,3 & 15,9 & 16,5 & 16,2 \\
Mariposita & 12,3 & 12,8 & 15,4 & 12,2 & 26,7 & 0,0 & 10,3 & 18,3 & 14,1 \\
Grama artificial & 13,8 & 4,3 & 2,6 & 2,0 & 0,0 & 0,0 & 4,0 & 7,0 & 5,4 \\
\hline Entrevistados & 65 & 47 & 39 & 49 & 30 & 11 & 126 & 115 & 241 \\
\hline
\end{tabular}


Foram propostas outras coberturas cuja função é mais ornamental (não suportam o pisoteio), e as seis espécies restantes variaram de 24,5 a $14,1 \%$ das respostas. A explicação para o maior favoritismo da grama pode ser a familiaridade dos moradores da cidade de Lima ao ver a maioria dos parques cobertos com grama e com algumas árvores, com pouca existência de outros tipos de cobertura. Também ajuda o fato de que a grama permite atividades sobre ela, sendo uma prática que os moradores estão acostumados.

As respostas também permitem inferir que os moradores entendem que os outros tipos de cobertura são apenas ornamentais e não podem ser calçadas, preferindo a grama pela resistência. Finalmente foi possível observar que poucos moradores $(5,4 \%)$ consideraram como uma opção a grama artificial, que mesmo tendo cores chamativas, não é do agrado dos entrevistados por ser um material sintético que acaba por romper com a ideia de parques naturais.

Quanto às faixas etárias, pode ser verificado que todas preferiram a grama natural, somando $72,7 \%$ no caso de 60 anos ou mais e $93,6 \%$ no grupo de 20 a 29 anos. É necessário ressaltar que a grama é um dos elementos que requer mais água em comparação com plantas de arbustos áridos, cítricos, frutíferos e ornamentais (DOMENE; SAURI, 2003), o que requer uma atenção em termos de manutenção.

Uma vez que praticamente inexistem parques públicos habilitados no La Encantada, procurou-se obter sugestões para a manutenção ao uso público. Logo, foi realizada uma pergunta de modo aberto a respeito da colaboração dos moradores em relação a seus parques. Pôde ser comprovado que as respostas mais frequentes estiveram relacionadas à limpeza (Tabela 9).

TABELA 8 - Como os moradores podem colaborar com a manutenção e cuidado de um parque? (\%)

\begin{tabular}{|c|c|c|c|c|c|c|c|c|c|}
\hline \multirow{2}{*}{ Respostas } & \multicolumn{6}{|c|}{ Faixa etária (anos) } & \multicolumn{2}{|c|}{ Gênero } & \multirow[t]{2}{*}{ Total } \\
\hline & $15-19$ & $20-29$ & 30-39 & $40-49$ & $50-59$ & $\geq 60$ & 우 & $0^{x}$ & \\
\hline Não sujando & 30,8 & 21,3 & 33,3 & 26,5 & 20,0 & 9,1 & 28,6 & 23,5 & 26,1 \\
\hline Quota econômica mensal & 27,7 & 23,4 & 12,8 & 26,5 & 30,0 & 9,1 & 26,2 & 20,9 & 23,7 \\
\hline Recolhendo as fezes dos cães & 21,5 & 10,6 & 10,3 & 20,4 & 10,0 & 9,1 & 15,1 & 15,7 & 15,4 \\
\hline Respeitando o regulamento & 1,5 & 4,3 & 17,9 & 10,2 & 13,3 & 9,1 & 9,5 & 7,0 & 8,7 \\
\hline Pagando tax & 0,0 & 12,8 & 10,3 & & 13,3 & 9,1 & 6,3 & 6,1 & 6,2 \\
\hline do as plantas & 4,6 & 6,4 & & & 6,7 & 0,0 & 6,3 & 4,3 & 5,4 \\
\hline Apoia & 4,6 & 6,4 & 7,7 & 4 & 6,7 & 0,0 & 2,4 & 8,7 & 5,4 \\
\hline Usan & 1,5 & 4,3 & 5,1 & 6 , & 6,7 & 9,1 & 4,8 & 4,3 & 4,6 \\
\hline Reco & 7,7 & 2,1 & 2,6 & 4, & 0,0 & 0,0 & 3,2 & 4,3 & 3,7 \\
\hline Apoia & 3,1 & 0,0 & 5,1 & 4 & 3,3 & 9,1 & 2,4 & 4,3 & 3,3 \\
\hline Regando os parques & 4,6 & 6,4 & 0,0 & 4,1 & 0,0 & 0,0 & 3,2 & 3,5 & 3,3 \\
\hline $\begin{array}{l}\text { Desenvolvendo sentido de } \\
\text { propriedade do mesmo }\end{array}$ & 1,5 & 4,3 & 2,6 & 0,0 & 3,3 & 18,2 & 1,6 & 4,3 & 2,9 \\
\hline Cuidar dos o & 7,7 & 0,0 & 0,0 & 2,0 & 3,3 & 0,0 & 1,6 & 4,3 & 2,9 \\
\hline Utiliz & 6,2 & 2,1 & 2,6 & 2,0 & 0,0 & 0,0 & 0,8 & 5,2 & 2,9 \\
\hline Oficir & 0,0 & 8,5 & 2,6 & 0,0 & 3,3 & 9,1 & 3,2 & 2,6 & 2,9 \\
\hline Outr: & 9,2 & 17,0 & 15,4 & 24,5 & 26,7 & 18,2 & 19,1 & 18,3 & 18,7 \\
\hline Não respondera & 3,1 & 2,1 & 2,6 & 2,0 & 3,3 & 0,0 & 3,2 & 1,7 & 2,5 \\
\hline Entrevistados & 65 & 47 & 39 & 49 & 30 & 11 & 126 & 115 & 241 \\
\hline
\end{tabular}

Sobre as maneiras pelas quais os moradores podem ajudar a cuidar dos parques, houve uma opinião majoritária em defesa da limpeza. Esse é um aspecto muito preocupante para os habitantes da cidade de Lima, a maioria das pessoas não colabora com a limpeza da cidade, deixando seus resíduos em vias públicas. Os 
moradores de La Encantada estão cientes disso e, portanto, invocam não sujar os parques. Outro aspecto recorrente foi o econômico: o pagamento de uma quota mensal esteve em segundo lugar nas respostas, tanto para homens como para mulheres; enquanto, de modo geral, a opção que sugeria que a manutenção dos parques deveria ser incluída nos pagamentos de impostos ao município esteve no quinto lugar. É interessante notar que, embora a maioria dos moradores queira uma área verde limpa, poucos expressam seu interesse em assumir a responsabilidade por fazê-lo. Isso confirma o que foi mencionado por De La Barrera et al. (2016a). Estes autores notaram que em bairros de baixa renda os habitantes da comunidade se sentem responsáveis por sua manutenção, enquanto nos bairros de classes mais altas (como La Encantada) tal responsabilidade é delegada inteiramente às autoridades municipais.

Tal como mencionado anteriormente, La Encantada é um bairro de alto poder aquisitivo, no entanto tem apenas um parque público em bom estado (com caminhos, manutenção da vegetação, limpeza e vigilância). Isto se reflete nesta pergunta, também de caráter aberto, em que tanto os homens quanto as mulheres mencionam como prioritário "ter mais áreas verdes" (19,8\% das mulheres e 15,7\% dos homens), atingindo uma média de 17,8\% (Tabela 10). As respostas seguintes também seguiram a mesma tendência: "fazer manutenção" $(5,4 \%)$, "deveriam melhorar os parques" (3,7\%) e "muito por trabalhar" $(3,7 \%)$, pode levar ao entendimento de que as áreas destinadas aos parques são percebidas como descuidadas.

TABELA 9 - Sugestões em relação aos parques de La Encantada (\%)

\begin{tabular}{|c|c|c|c|c|c|c|c|c|c|}
\hline \multirow{2}{*}{ Sugestões } & \multicolumn{6}{|c|}{ Faixa etária (anos) } & \multicolumn{2}{|c|}{ Gênero } & \multirow{2}{*}{ Total } \\
\hline & $15-19$ & $20-29$ & $30-39$ & $40-49$ & $50-59$ & $\geq 60$ & 우 & $0^{\pi}$ & \\
\hline Ter mais áreas verdes & 15,4 & 17,0 & 25,6 & 18,4 & 16,7 & 9,1 & 19,8 & 15,7 & 17,8 \\
\hline Fazer manutenção & 9,2 & 8,5 & 0,0 & 6,1 & 0,0 & 0,0 & 5,6 & 5,2 & 5,4 \\
\hline $\begin{array}{l}\text { Deveriam melhorar os } \\
\text { parques }\end{array}$ & 6,2 & 2,1 & 0,0 & 4,1 & 3,3 & 9,1 & 4,0 & 3,5 & 3,7 \\
\hline Muito por trabalhar & 1,5 & 2,1 & 7,7 & 6,1 & 3,3 & 0,0 & 3,2 & 4,3 & 3,7 \\
\hline $\begin{array}{l}\text { Ter plantas apropriadas à } \\
\text { área }\end{array}$ & 3,1 & 0,0 & 2,6 & 8,2 & 3,3 & 0,0 & 4,0 & 2,6 & 3,3 \\
\hline Não há bons parques & 1,5 & 8,5 & 0,0 & 2,0 & 3,3 & 0,0 & 3,2 & 2,6 & 2,9 \\
\hline Jogo & 3,1 & 0,0 & 5,1 & 4 & 0,0 & 0,0 & 0,8 & 4,3 & 2,5 \\
\hline Pôr vi & 1,5 & 2,1 & 0,0 & 2,0 & 0,0 & 27,3 & 2,4 & 2,6 & 2,5 \\
\hline Podem ter melhor cuidado & 1,5 & 2,1 & 0,0 & 2,0 & 0,0 & 9,1 & 2,4 & 0,9 & 1,7 \\
\hline $\begin{array}{l}\text { Formar um comite de } \\
\text { moradores }\end{array}$ & 0,0 & 2,1 & 2,6 & 0,0 & 3,3 & 9,1 & 1,6 & 1,7 & 1,7 \\
\hline Rapidez em fazer os parques & 1,5 & 0,0 & 5,1 & 0,0 & 3,3 & 0,0 & 2,4 & 0,9 & 1,7 \\
\hline $\begin{array}{l}\text { Recolher os dejetos dos } \\
\text { animais de estimação }\end{array}$ & 1,5 & 2,1 & 0,0 & 2,0 & 3,3 & 0,0 & 2,4 & 0,9 & 1,7 \\
\hline $\begin{array}{l}\text { Regar os parques mais } \\
\text { continuamente }\end{array}$ & 4,6 & 0,0 & 0,0 & 0,0 & 0,0 & 0,0 & 1,6 & 0,9 & 1,2 \\
\hline Lixeiras & 0,0 & 2,1 & 0,0 & 2,0 & 3,3 & 0,0 & 1,6 & 0,9 & 1,2 \\
\hline Outra & 6,1 & 12,8 & 7,7 & & 36,7 & 63,6 & 20,6 & 15,6 & 18,2 \\
\hline Não & 47,7 & 44,7 & 48,7 & 34,7 & 33,3 & 27,3 & 38,9 & 45,2 & 41.9 \\
\hline Entrevistados & 65 & 47 & 39 & 49 & 30 & 11 & 126 & 115 & 241 \\
\hline
\end{tabular}

Quanto aos grupos de idades, todos, com exceção da faixa de 60 anos ou mais, mencionaram como prioritário ter mais áreas verdes. O grupo de 60 anos ou mais considerou como prioritário "pôr vigilância", colocando em segundo lugar "ter áreas verdes". Importante observar o alto número de outras respostas mencionadas, 
manifestando assim um interesse dos moradores na existência de áreas verdes adequadas que possam ser utilizadas por todos. Finalmente, a Tabela 11 apresenta a distribuição de faixa etária por gênero dos 241 entrevistados.

TABELA 10 - Distribuição por faixa etária por gênero

\begin{tabular}{ccccccc}
\hline \multirow{2}{*}{$\begin{array}{c}\text { Faixa etária } \\
\text { (anos) }\end{array}$} & \multicolumn{2}{c}{ Feminino } & \multicolumn{2}{c}{ Masculino } & \multicolumn{3}{c}{ Total } \\
\cline { 2 - 7 } & $\mathbf{N}$ & $\%$ & $\mathbf{N}$ & $\%$ & $\mathbf{N}$ & $\%$ \\
\hline $15-19$ & 31 & 24,6 & 34 & 29,6 & 65 & 27,0 \\
$20-29$ & 22 & 17,5 & 25 & 21,7 & 47 & 19,5 \\
$30-39$ & 21 & 16,7 & 18 & 15,7 & 39 & 16,2 \\
$40-49$ & 32 & 25,4 & 17 & 14,8 & 49 & 20,3 \\
$50-59$ & 14 & 11,1 & 16 & 13,9 & 30 & 12,4 \\
$\geq 60$ & 6 & 4,8 & 5 & 4,3 & 11 & 4,6 \\
\hline Total & 126 & 100 & 115 & 100 & 241 & 100 \\
\hline
\end{tabular}

*As diferenças entre gêneros e idades não são significativas.

Procurou-se amostrar proporcionalmente entre homens e mulheres e entre as diferentes faixas etárias. Os resultados encontrados foram semelhantes ao encontrado por Menezes (2011), que obteve proporção de $52 \%$ de mulheres e $48 \%$ de homens e com pouco mais de metade da amostra $(57,67 \%)$ composta por pessoas acima de 34 anos.

O tamanho amostral utilizado para a realização dos questionários foi adequado para obter as respostas necessárias para analisar a percepção dos moradores de La Encantada sobre as áreas verdes. Uma alta coincidência foi encontrada nas respostas para a maioria das questões. Percebe-se claramente o interesse dos moradores em ter áreas verdes públicas, com o objetivo principal de ter um ponto de encontro, um local de brincadeiras para as crianças e que o bairro tenha locais com vegetação. O uso de imagens para descobrir as preferências estéticas dos moradores em relação às árvores, arbustos e cobertura do solo é inovador, mas há sempre o risco de que a qualidade da foto possa influenciar as respostas. No entanto, uma ideia clara foi obtida das preferências dos moradores em relação aos tipos de vegetação que preferem. Por fim, o trabalho realizado sobre a questão da participação da população, sobre a responsabilidade pelo cuidado das áreas verdes e a gestão de resíduos, mostra claramente a disposição dos moradores em colaborar para ter espaços verdes permanentes para o desfrute da comunidade.

\section{CONCLUSÕES}

Os resultados deste estudo mostram que a grande maioria da população do bairro La Encantada, especialmente aqueles com mais de 40 anos, considera as áreas verdes públicas como muito importantes para o local.

As respostas obtidas mostram que, embora a maioria dos habitantes do local tenha clubes e jardins privados, eles valorizam a existência desses espaços para apreciar a natureza, o prazer das crianças e o encontro com os vizinhos. No entanto, estudos futuros devem tentar entender a razão pela qual, embora os moradores considerem as áreas verdes importantes, o bairro tem apenas um dos dez espaços para parques totalmente habilitados.

O uso do questionário feito aos moradores ajudou a obter resultados concretos quanto às preferências sobre o tipo de vegetação que as áreas deveriam ter, bem como a infraestrutura de apoio. É aconselhável realizar pesquisas em bairros de outros níveis socioeconômicos para contrastar opiniões. 


\section{REFERÊNCIAS}

AGUILAR, M.; MERCON, J.; SILVA, E. Aportaciones de las percepciones socioecológicas a la Educación Ambiental. Entreciencias, v. 5, n. 15, p. 95-110, 2017. DOI: https://dx.doi.org/10.22201/enesl.20078064e.2017.15.62581

APEIM - Asociación Peruana de Empresas de Investigación de Mercados. Niveles socioeconómicos 2017. Disponível em: < http://www.apeim.com.pe/wpcontent/themes/apeim/docs/nse/APEIM-NSE-2017.pdf>. Acesso em: set. 2018.

BARDIN, L. Análise de conteúdo. Lisboa: Edições 70, 1980, 229 p.

CALIXTO, R.; HERRERA, L. Estudo sobre as percepções e a educação ambiental. Tempo de Educar, Universidade Autônoma do Estado de México, n. 11, v. 22, p. 227-249, 2010.

CARRUS, G.; SCOPELLITI, M.; LAFORTEZZA, R.; COLANGELO, G.; FERRINI, F.; SALBITANO, F.; AGRIMI, M.; PORTOGHESI, L.; SEMENZATO, P.; SANESI, G. Go greener, feel better? The positive effects of biodiversity on the well-being of individuals visiting urban and peri-urban green areas. Landscape and Urban

Planning, $\quad$ v. $134, \quad$ p. 221-228, $2015 . \quad$ DOI: https://doi.org/10.1016/j.landurbplan.2014.10.022

DE LA BARRERA, F.; REYES-PAECKEB, S.; HARRIS, J.; BASCUÑAN, D.; FARIAS, J.M. People's perception influences on the use of green spaces in socioeconomically differentiated neighborhoods. Urban Forestry \& Urban Greening, v. 20, p. 254-264, 2016a. DOI: https://doi.org/10.1016/j.ufug.2016.09.007

DE LA BARRERA, F.; REYES-PAECKEB, S.; BANZHAF, E. Indicators for green spaces in contrasting urban settings. Ecological Indicators, v. 62, p. 212-219, 2016b. DOI: https://doi.org/10.1016/j.ecolind.2015.10.027

DOMENE, E.; SAURI, D. Modelos urbanos y consumo de agua: el riego de jardines privados en la región metropolitana de Barcelona. Investigaciones Geográficas, Universidad de Alicante, n. 32, p. 5-17, 2003. DOI: http://dx.doi.org/10.14198/INGEO2003.32.02

FENG, X; ASTELL-BURT, T. Residential green space quantity and quality and child well-being: a longitudinal study. American Journal of Preventive Medicine, v. 53, n. 5, p. 616-624, 2017. DOI: 10.1016/j.amepre.2017.06.035.

GOMES RUBIRA, F. Definição e diferenciação dos conceitos de áreas verdes/espaços livres e degradação ambiental/impacto ambiental. Caderno de Geografia, v. 26, n. 45, p. 134-150, 2016. DOI: https://doi.org/10.5752/P.23182962.2016v26n45p134

GOOGLE EARTH. Disponível em: <https://www.google.com/earth/>. Acesso em: Novembro 2016. 
INSTITUTO NACIONAL DE ESTADÍSTICA Y GEOGRAFÍA - MÉXICO. Diseño de cuestionarios. INEGI. 2013. 61 p.

KRELLENBERG, K.; WELZ, J.; REYES-PACKE, S. Urban green areas and their potential for social interaction: a case study of a socio-economically mixed neighbourhood in Santiago de Chile. Habitat Internacional, v. 44, p. 11-21, 2014. DOI: https://doi.org/10.1016/j.habitatint.2014.04.004

MADUREIRA, H.; NUNES, F.; VIDAL OLIVEIRA, J., CORMIER, L.; MADUREIRA, T. Urban residents' beliefs concerning green space benefits in four cities in France and Portugal. Urban Forestry \& Urban Greening, v. 14, p. 56-64, 2015. DOI: https://doi.org/10.1016/j.ufug.2014.11.008

MADUREIRA, H.; NUNES, F.; VIDAL OLIVEIRA, J., MADUREIRA, T. Preferences for urban green spaces characteristics: a comparative study in three Portuguese cities. Environments, v. 25, n. 5, p. 1-13, 2018. DOI: https://doi.org/10.3390/environments5020023

MARTINEZ-SOTO, J; MONTERO Y LÓPEZ LENA, M.; DE LA ROCA, J. M. Efectos psicoambientales de las áreas verdes en la salud mental. Interamerican Journal of Psychology, v. 50, n. 2, p. 204-214, 2016. Disponível em: <http://www.redalyc.org/articulo.oa?id=28447010004>.

MENEZES, J.P.C. Percepção ambiental dos visitantes do Parque Municipal Bosque John Kennedy - Araguari, MG. Revista Eletrônica do Mestrado em Educação Ambiental, Rio Grande, n. 26, p. 103-112., $2011 . \quad$ DOI: $<$ https://doi.org/10.14295/remea.v26i0.3349>.

NAÇÕES UNIDAS. Relatório da conferência das Nações Unidas sobre os assentamentos humanos (HABITAT II), 3 ao 14 de junho de 1996. Istambul, $250 \mathrm{p}$.

SCHÄFFLER, A.; SWILLING, M. Valuing green infrastructure in an urban environment under pressure - the Johannesburg case. Ecological Economics journal, v. 86, p. 246-257, 2013. DOI: https://doi.org/10.1016/j.ecolecon.2012.05.008

SZEREMETA, B.; ZANNIN, P.H.T. A importância dos parques urbanos e áreas verdes na promoção da qualidade de vida em cidades. Ra'ega. Espaço Geográfico em Análise, n. 29, p. 177-193, 2013. DOI: http://dx.doi.org/10.5380/raega.v29i0.30747.

WOLCH, J.; BYRNE, J.; NEWELL, J. Urban green space, public health, and environmental justice: the challenge of making cities 'just green enough'. Landscape and Urban Planning, v. 125, p. 234-244, 2014. DOI: https://doi.org/10.1016/j.landurbplan.2014.01.017 